\title{
Movilización del conocimiento en educación inclusiva: conceptualización, roles y estrategias
}

\author{
Knowledge mobilization in inclusive education: \\ conceptualization, roles and strategies
}

\author{
Cristina Gimeno-Pérez, Paula Escobedo-Peiro ${ }^{* *}$
}

Recibido: 23 de noviembre de 2020 Aceptado: 23 de diciembre de 2020 Publicado: 27 de julio de 2021

To cite this article: Gimeno Pérez, C. y Escobedo Peiro, P. (2021). Movilización del conocimiento en educación inclusiva: conceptualización, roles y estrategias. Márgenes, Revista de Educación de la Universidad de Málaga. 2 (2), 25-41

DOI: https://doi.org/10.24310/mgnmar.v2i2.10892

\section{RESUMEN}

Este estudio se centra en la Movilización Del Conocimiento (MDC) y su vinculación en el contexto de la investigación inclusiva. Metodológicamente, el trabajo se desarrolla desde un enfoque cualitativo-descriptivo para identificar la conceptualización del término, los condicionantes, roles, estrategias y procesos intragrupales que desarrolla un grupo de investigadores que trabajan en educación inclusiva. Tras la realización de un grupo focal con estos investigadores para indagar sobre cómo ellos entienden la MDC y cómo la trabajan, se analizó la información mediante el análisis de contenido y se establecieron categorías teóricas e inductivas (emergentes del análisis). Algunos resultados apuntan que estos investigadores trabajan la MDC desde un paradigma de investigación participativa e inclusiva; aspiran a coinvestigar para favorecer una participación más democrática y los plazos de tiempo, en este tipo de investigación, pueden ser una condición limitadora. La investigación concluye estableciendo orientaciones y futuras vías de investigación relacionadas con la MDC.

Palabras clave: movilización del conocimiento; roles y estrategias; investigación inclusiva

\section{ABSTRACT}

This study inquires about knowledge mobilization $(\mathrm{KMb})$ and its connection with inclusive investigation. Methodologically, the study is developed from the qualitative-descriptive research approach, trying to identify the terminology, the conditions, the roles, strategies and intragroup processes which a group of researchers in inclusive investigation. After develop a focal group with these investigators to investigate how they understand and carry out the $\mathrm{KMb}$, the analysis of the information was carried out using the content analysis technique and were established theoretical and inductive categories (emerging from the analysis). Some results indicate that these researchers mobilize knowledge from a paradigm of participative and inclusive investigation, they aspire to coinvestigate to favor a more democratic participation, and the terms of time of this type of research can be a limiting condition. The research concludes trying to stablish guidelines and future research paths of KMb.

Keywords: knowledge mobilization; roles and strategies; inclusive investigation 


\section{INTRODUCCIÓN}

Dar respuesta a la diversidad del alumnado, de manera equitativa y justa, es uno de los mayores desafíos y obligaciones que debe atender la educación. Para lograrlo, es necesaria una forma de investigar que analice y reflexione sobre el papel y las actuaciones que se llevan a cabo en la comunidad educativa. Esto supone desarrollar procesos participativos y democráticos por y para el derecho a una educación inclusiva (Echeita, 2017).

En esta línea, Barrio (2009) identifica la educación inclusiva como la clave política para la construcción de la sociedad que deseamos. El sistema educativo no puede ser el epicentro de exclusiones sociales, económicas y culturales, sino que debería ser el modelo educativo que implicara la participación ciudadana, democrática y la innovación social para reconocer y atender las necesidades e intereses con la finalidad de avanzar hacia una sociedad sin barreras (Pérez, García y Ortiz, 2016).

Además de esto, es necesario revisar la práctica educativa, en concreto las estrategias didácticas que se llevan a cabo para el desarrollo inclusivo del conocimiento. Para ello, es importante que se indague desde las investigaciones sobre el vínculo entre teoría y práctica, también desde la mirada de los propios agentes. En este sentido, Ander-Egg (2003) propone la InvestigaciónAcción-Participativa (IAP) como método de investigación, desde el que abordar la transformación y la mejora social para la inclusión. Señala que la IAP tiene el propósito de estudiar y resolver problemas de interés práctico; cuestiones significativas para un determinado grupo o colectivo de personas que desean actuar para superar las barreras y desarrollarse en un entorno inclusivo. La IAP también valora los resultados obtenidos de las investigaciones que se basan en las voces de los participantes. Esta idea es ampliada por Levin (2008), que identifica la Movilización del conocimiento en la relación que se da entre investigación y práctica.

La comunicación sobre el aprendizaje construido que surge de las investigaciones y de las acciones participativas, da lugar al término movilización del conocimiento "knowledge mobilization" (MDC), acuñado por el Consejo de Investigación de Ciencias Sociales y Humanidades de Canadá (SSHRC). Desde la Universidad de Quebec en Montreal (UQAM), indican que la movilización del conocimiento es el conjunto de procesos que tienen como objetivo intercambiar conocimiento (investigación, prácticas, experiencias y culturas) con el objetivo de crear nuevas formas que puedan utilizarse para la acción.

De modo que, movilizar el conocimiento no significa divulgar información de forma unidireccional, sino que hace referencia a la amplia gama de conocimiento producido, investigado y difundido entre todos los agentes educativos e investigadores, beneficiando a toda la comunidad y pudiendo mostrar impactos positivos de manera internacional. Es un proceso en el que necesariamente, tiene lugar el diálogo entre todas las voces y agentes; el intercambio de visiones respecto a los conocimientos que se tratan y la capacidad de expresar las ideas en diferentes contextos. En esencia, no se pierde de vista el objetivo inclusivo y participativo que lo define, sino que se demuestra que el conocimiento y el aprendizaje están vivos y son proclives a compartirse y a completarse mediante la interacción. La MDC trabaja también la accesibilidad de los saberes. Puesto que su tratamiento es móvil, no queda encasillado en un entorno o público específico o a través de un vocabulario excluyente e incorpora la participación de toda la comunidad. 
Sin embargo, actualmente es difícil identificar la MDC debido a la falta de investigación sobre el funcionamiento de las conexiones y redes cooperativas entre investigadores, docentes, familias, alumnado y del propio entorno (Moliner y Ramel, 2018). Cabe destacar que las relaciones entre los agentes son fundamentales, tanto para crear redes cooperativas de conocimiento como para identificar los roles, estrategias y prácticas que se desempeñan y los condicionantes que existen para conseguir la MDC.

De acuerdo con Landry et al. (2008), la visión y labor de los equipos investigadores sobre MDC es importante para lograr cambios que se persiguen también desde la IAP. En este tipo de investigaciones, los investigadores están al servicio de la comunidad y desempeñan funciones para establecer vínculos en los que la comunidad se sienta cómoda. Enlazando esto con los diferentes roles en los que se posiciona el investigador dentro de un proceso de IAP, destacamos la categorización que realizan Wang y Mu (2013). En concreto, en su trabajo sitúan los roles de los investigadores en el proceso de Investigación-Acción (IA). Identifican el rol de instructor y experto en la etapa introductoria; añaden el rol de facilitador, patrocinador y proveedor de recursos durante la planificación; el de observador, oyente y aprendiz durante la implementación; el impulsor y cuidador afectivo durante el análisis y evaluación de los datos, y finalmente el rol de editor y coautor de artículos hacia el final del proyecto.

Por su parte, Dolbec y Prud'homme (2009), inciden en la orientación hacia la tarea inclusiva y emancipadora de la investigación, identificando cuatro roles: investigador profesional, formador, facilitador del trabajo colaborativo y agente de cambio. De este modo, adentrarnos en roles que hacen más accesible la información y el intercambio de conocimiento parte del deseo de romper con barreras infranqueables que se ciñen a establecer relaciones unidireccionales.

En contraposición, la MDC necesita instrumentos y técnicas inclusivas para generar espacios participativos. El SSHRC (2017) remarca instrumentos y técnicas accesibles para utilizar en la MDC, como libros; videos; repositorios en línea; etc. Sin embargo, la MDC también necesita estrategias. Acciones encaminadas a considerar la pluralidad de los contextos en los que cada persona convive, para ofrecer espacios en los que haya lugar a la convivencia democrática y a la participación activa de la sociedad. De ahí que, Parrilla, Raposo-Rivas y Martínez-Figueira (2016), hablen de estrategias que faciliten la comunicación. Aunque, además de estas, es necesario trabajar el empoderamiento, como "uno de los objetivos prioritarios en cualquier proceso de transformación” (Traver, Sales y Moliner, 2010, p.107).

Por otro lado, en el ámbito de esta investigación es esencial conocer las condiciones que facilitan o dificultan la MDC en investigaciones sobre inclusión educativa. Por una parte, Levin (2008), considera que la ausencia de una representación común que defina la MDC, en contraposición de una gran variedad de terminologías y de marcos conceptuales, conlleva al solapamiento de significados sobre el término. Así como Fischman, Anderson y Zuiker (2018) hablan de la disparidad entre las organizaciones y los procedimientos para llevar la MDC a cabo y señalan que "la movilización del conocimiento en gran medida se considera que está fuera de las políticas de titularización y de promoción” (p. 9).

Por otra parte, el SSHRC identifica como pauta facilitadora que los equipos investigadores demuestren una actitud activa en el desarrollo de la MDC. En esta línea, la divulgación de los 
resultados obtenidos en las investigaciones, así como de los procesos vividos, es una condición sine qua non para la MDC. Pero, no todos los medios son ideales, sino que han de poseer un enfoque reflexivo, para que la mayor parte de la población llegue a construir nuevos conocimientos y puedan interiorizarlos y utilizarlos en futuras ocasiones.

En el artículo que presentamos, pretendemos analizar el concepto, los roles y estrategias sobre la MDC. Concretamente, nos centraremos en un equipo de investigación, que mayormente investiga sobre procesos de transformación hacia la inclusión educativa en escuelas. Este trabajo, parte del proyecto de $I+D$ "Movilización del conocimiento sobre Educación Inclusiva: Roles y Estrategias de los investigadores/formadores en procesos de mejora escolar desde la investiga-

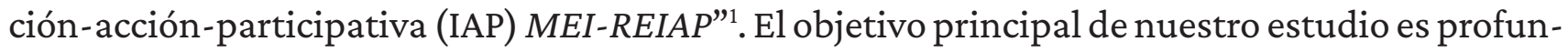
dizar sobre cómo la MDC favorece una educación inclusiva. Para ello, planteamos las siguientes preguntas: ¿qué significa la MDC en educación inclusiva, para estos investigadores?, ¿cuáles son los roles que ejercen?, ¿qué estrategias llevan a cabo?, ¿qué condiciones facilitan o dificultan la MDC?, y ¿qué procesos se realizan dentro del grupo para favorecer la MDC?

\section{MÉTODO}

La investigación se enmarca en un diseño cualitativo, de tipo descriptivo que se lleva a cabo desde una perspectiva fenomenológica para determinar e indagar el significado y la forma en cómo los participantes describen experiencias sobre un hecho determinado (Bisquerra, 2004).

\subsection{Participantes}

Los participantes del proyecto de investigación autonómico en el que se enmarca este estudio son investigadores de distintas universidades españolas, que tienen en común investigar sobre inclusión y desde un enfoque inclusivo. Por ello, hablamos de una muestra incidental. En el caso del trabajo que presentamos, la muestra se focalizó en uno de estos grupos de discusión. Se contó con la participación de ocho informantes, todos ellos investigadores del ámbito de la educación inclusiva. Siete profesores universitarios y una colaboradora del grupo, estudiante de Doctorado. Todos con edades comprendidas entre los 29 y los 57 años. Entre estos, un informante hombre y siete mujeres.

\subsection{Recogida de la información e instrumento}

La información fue recabada mediante un grupo focal y su respectivo retorno de la información. La dinamizadora de las sesiones propuso una serie de preguntas respecto a la MDC con el fin de que surgieran actitudes, sentimientos, creencias, experiencias y reacciones en los participantes (Bonilla-Jiménez y Escobar, 2017) que ayudaran a definir nuestro objeto de estudio.

En las dos sesiones participaron los ocho informantes del grupo de investigación. Se estableció un clima de acogida donde tuvieron lugar todas las voces, las preguntas fueron formuladas

1 Proyecto dirigido por la Dra. Odet Moliner García, dentro de la Convocatoria de Subvenciones para Grupos de Investigación Consolidables 2018 (AICO18/066) de la Generalitat Valenciana DOCV 07-11-2017 N8164 
de forma abierta y general y las intervenciones fueron dinamizadas a modo de debate. En ambas reuniones las respuestas fueron registradas mediante grabaciones de audio, para la transcripción y análisis de la información obtenida, considerando el principio de confidencialidad, ya que los datos son tratados desde el anonimato.

Cada sesión tuvo una duración aproximada de una hora. Antes del grupo focal se procedió a la firma del consentimiento informado, así como al permiso de grabación con los datos sociodemográficos de los participantes donde también aparecían el objetivo y las preguntas de investigación (de acuerdo con el protocolo de actuación establecido como instrumento del proyecto autonómico). Dos semanas después se realizó el retorno, donde se procedió a la devolución de los datos extraídos del grupo focal y se trataron informaciones que necesitaban ser clarificadas.

\subsection{Procedimiento de codificación y análisis de datos}

En cuanto a la codificación de las unidades de significado, se asigna a cada una la referencia del grupo focal (GF) o del retorno (R)y a cada informante se le asigna un código (I1 hasta I8).

El análisis de la información se efectuó de manera deductiva e inductiva. Para ello, se transcribieron aquellas partes más relevantes de las grabaciones del GF y del R, en lugar de realizar una transcripción literal, puesto que este tipo de transcripción ofrece una mayor inmediatez de la información para su análisis. A su vez, se escribieron, a modo de "memos", pequeñas anotaciones interpretativas sobre los datos y posteriormente se realizó un análisis de contenido.

Se realizó una categorización deductiva organizando los datos en las cinco categorías teóricas correspondientes a las temáticas principales de las preguntas de investigación (definición del concepto, roles, estrategias, condicionantes y proceso intragrupal que seguía el grupo para la MDC). Posteriormente, este análisis se completó con la emergencia de categorías inductivas. Así pues, las categorías se sustentan en los datos extraídos de las aportaciones de los informantes y responden a las preguntas de investigación.

A continuación, establecimos relaciones entre las categorías, en función de los datos que sustentaban las mismas, a partir de mapas conceptuales mediante la herramienta informática Cmap Tools.

\section{RESULTADOS}

Los resultados se estructuran en función de las preguntas planteadas en la investigación. A continuación, se describen y organizan las categorías definidas, considerando los porcentajes de aportaciones realizadas por los participantes en cada una de las categorías: a) Definición del concepto; b) Roles; c) Estrategias; d) Condicionantes; e) Proceso intragrupal para la MDC (según vemos en la Tabla 1 sobre categorías y porcentajes). 
maroenes

\section{N V E S T I G A C I O N E S}

Tabla 1. Porcentaje de unidades de análisis por categorías. Fuente: elaboración propia

\begin{tabular}{cc}
\hline CATECORÍAS & Porcentajes de citas \\
\hline Definición del concepto & $12.82 \%$ \\
\hline Roles & $33.33 \%$ \\
\hline Estrategias & $20.51 \%$ \\
\hline Condicionantes & $12.82 \%$ \\
\hline Proceso intragrupal para la MDC & $20.51 \%$ \\
\hline
\end{tabular}

En líneas generales se observa que los participantes hacen más referencia a los roles empleados (33.3\%), estrategias (20.51\%) y el proceso intragrupal $(20.51 \%)$ que a la definición del concepto (12.82\%) y a los condicionantes (12.82\%).

a. Conceptualización de la movilización del conocimiento de la investigación sobre educación inclusiva

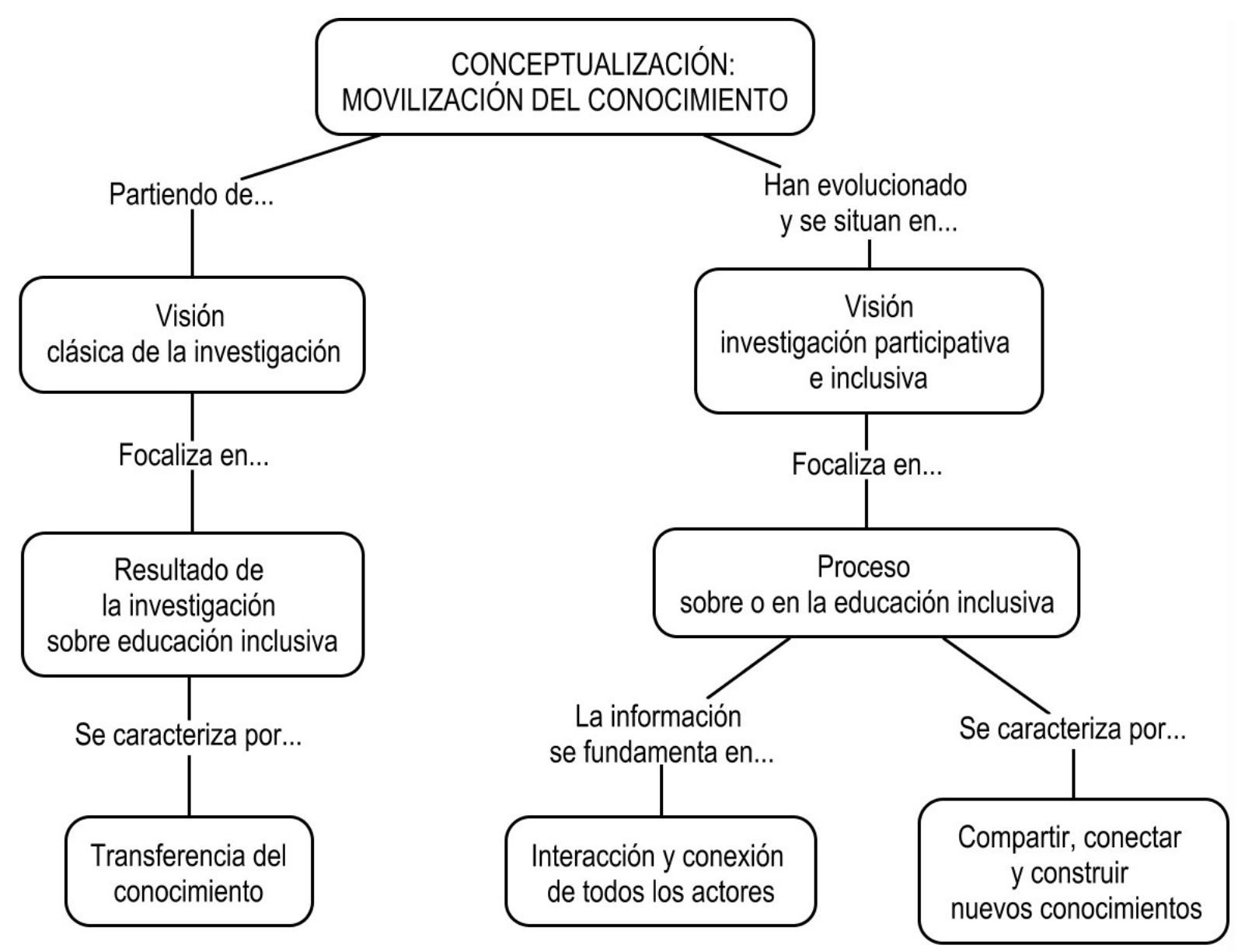

Gráfico 1. Resultados sobre la conceptualización de movilización del conocimiento. Fuente: Elaboración propia 


\section{N V E S T I G A C I O N E S}

En primer lugar, dentro del término MDC, los participantes identifican un continuum entre una visión más clásica de la investigación y otra más participativa e inclusiva. Aunque, reconozcan la visión más clásica, se sitúan claramente dentro de un paradigma de investigación participativo e inclusivo. Consideran que, han partido de una visión más clásica (más centrada en transferir información), y han podido evolucionar hacia una visión de investigación participativa e inclusiva. "Hay una visión de investigación participativa que focaliza más sobre el proceso y otra más clásica que focaliza sobre los resultados.” (I1, R).

Aunque resaltan la importancia de atender ambas perspectivas, consideran que, la visión anterior se centra en los resultados y que a día de hoy están más interesados en el proceso de investigación. En ese mismo sentido, añaden que la MDC es una forma de compartir saberes, pues "movilizar se utiliza en el sentido de construir conocimiento conjuntamente, compartirlo y después divulgarlo, difundirlo. Desde una relación más de interacción entre los actores.” (I2, GF). Y también lo asocian a una manera de construir nuevos conocimientos, de manera participativa, dando lugar a todas las voces "el conocimiento se genera, se construye de una manera determinada y suelen ser investigaciones participativas donde se democratiza todo ese proceso y se da acceso a otras personas, a otras voces.” (I3, GF).

Además, resaltan la función que ejerce la MDC en conectar y hacer accesibles los saberes, "La MDC también va en este sentido: en cómo encaja nuestro marco teórico o nuestra fundamentación con lo que se trabaja en la escuela.” (I4, GF). Y otro aspecto en el que inciden es que la MDC es un proceso que se fomenta y sustenta por el empoderamiento de las personas. Ya que esto consiste en generar conocimientos que produzcan transformaciones, tanto individuales como sociales. Pues, "todos los saberes, no solo los de los investigadores, son importantes y están legitimados para construir nuevos saberes. [...]. En definitiva, hablamos de un conocimiento que empodera, que ayuda a transformar realidades.” (I1, GF).

b. Roles asumidos por los investigadores para realizar la investigación en educación inclusiva

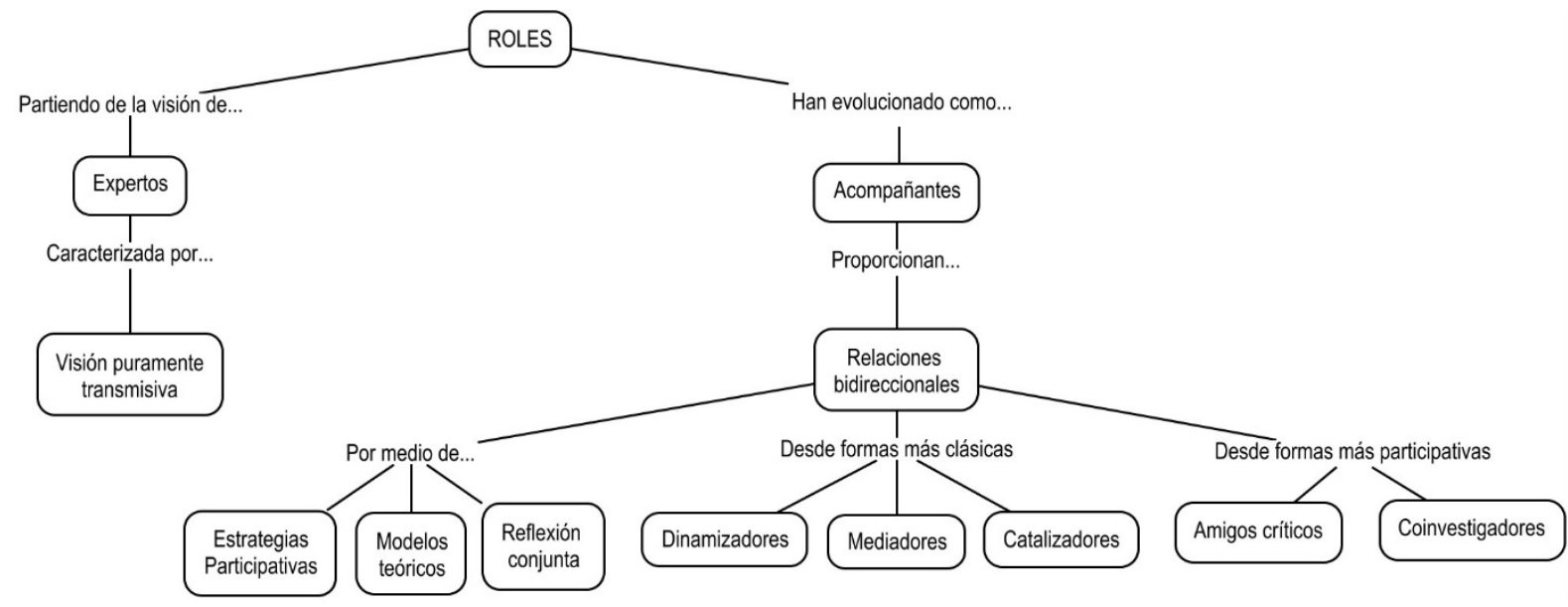

Gráfico 2. Resultados sobre los roles en la movilización del conocimiento. Fuente. Elaboración propia 
$\mathrm{Al}$ visualizar la MDC como un proceso, los informantes incorporan rápidamente en su discurso los roles que ejercen para llevarla a cabo. Así pues, en esta segunda pregunta, identifican un continuum entre la visión de expertos y la de acompañantes.

Los investigadores consideran que han partido de una figura o rol de experto desde el cual continúan evolucionando hacía un rol de acompañante. "El grupo ha tenido que romper con una estructura que es la de la universidad y el de ir como expertos para poder ir a ese camino de acompañamiento y adoptar ese rol. Esto no ha sido automático." (I4, GF).

Además, destacan que esta evolución ha sido fruto de la propia reflexión sobre los procesos de investigación, puesto que "ha habido más aprendizaje del proceso que del producto." (I4, GF). La informante 3 opina que las necesidades del contexto, en ocasiones, les hacen adoptar una figura de experto como persona externa que aporta información que por su posición pueden conocer y que el resto de los participantes no. Y el informante 1 coincide en que llevar a cabo este rol es sinónimo de actuar para transmitir y proporcionar información, aunque lo clasifique: "como medio para llegar a otro lugar, no para quedarnos ahi." (I1, GF).

Por otra parte, el rol de acompañante, es aquel en el que se sitúan. Donde establecen relaciones bidireccionales con el resto y se ponen al servicio de la mejora del contexto. Ya que el tipo de investigación que practican, requiere de este acompañamiento. Tal y como expresa la Informante 2: "Yo me veo acompañando al centro, con una mente muy abierta. [...]. Es algo bidireccional y te sitúas en ese rol de ponerte al lado de..." (I2, GF). Y para poder llevar a cabo este rol la Informante 3 destaca el uso y proporción de "estrategias participativas, modelos teóricos y reflexión conjunta." (I3, GF).

Además, dentro de este rol de acompañante ubican varios roles que interactúan entre sí y que ayudan a establecer estas relaciones bidireccionales con los agentes sociales. "Dinamizadores, mediadores y catalizadores son formas de acompañar más clásicas.” (I.1, R). Aquí visualizan el rol de dinamizador, proporcionando situaciones nuevas "a veces somos dinamizadores, generamos situaciones que no se habían dado y que no están en la estructura cotidiana de esa organización o ese grupo de personas." (I3, GF), así como ocurre con el rol de mediador, el cual identifican con proporcionar ajustes necesarios dentro los procesos de investigación. No obstante, partiendo de que el mediador interviene para proporcionar accesibilidad, los informantes 3 y 1 destacan que este rol confluye con la denominación de catalizador.

"El mediador me parece interesante cuando generamos espacios de reflexión, para compartir, para analizar, para hacer una técnica de espejo y compartir información, o para trabajar un concepto en el claustro [...]. A veces se explicita que necesitas que venga alguien para que haga la pregunta que tú no te atreves a hacer". (I3, GF)

Por otra parte, dentro del rol de acompañante se ubican también formas más participativas, por ejemplo "el amigo crítico va más a provocar. [...] Sobre todo a problematizar." (I1, R). También, el rol de coinvestigador, relacionado con la solidaridad, la colaboración y las relaciones basadas en la igualdad: "a veces es un rol de igual, de coinvestigador, de persona que se hace la misma pregunta y trata de buscar la respuesta por algún camino.” (I3, GF1). Adoptar el rol de coinvestigador los acerca todavía más al paradigma desde el que parten, "en todos los momen- 


\section{N V E S T I G A C I O N E S}

tos de la investigación no creo que coinvestiguemos, pero sí que es una aspiración, porqué creo que es muy significativo para la investigación participativa." (I2, GF). Aunque, comentan que coinvestigar no es tarea fácil (I5, GF).

Coinciden en que pueden practicar estos roles: experto, acompañantes (dinamizadores, mediadores, catalizadores, amigos críticos, coinvestigadores), ajustándose a las exigencias, necesidades y tiempos del proceso de investigación. Destacan los ritmos como un aspecto determinante para adoptar un rol u otro: "A veces vas con un rol determinado, pero en el momento en el que se encuentran te exigen otro, porque necesitan otro. [...], los tiempos no son los mismos y a veces hay que ajustarse." (I4, GF). Y ante esto, apuestan por la flexibilidad durante el proceso, "van apareciendo cosas que te hacen ajustarte a que puedas situarte más [...]. Es decir, tú te vas ajustando en el momento de la investigación.” (I5, GF), y para ello son indispensables una serie de estrategias.

\section{c. Estrategias que se emplean para movilizar el conocimiento en el contexto de investi- gación y formación}

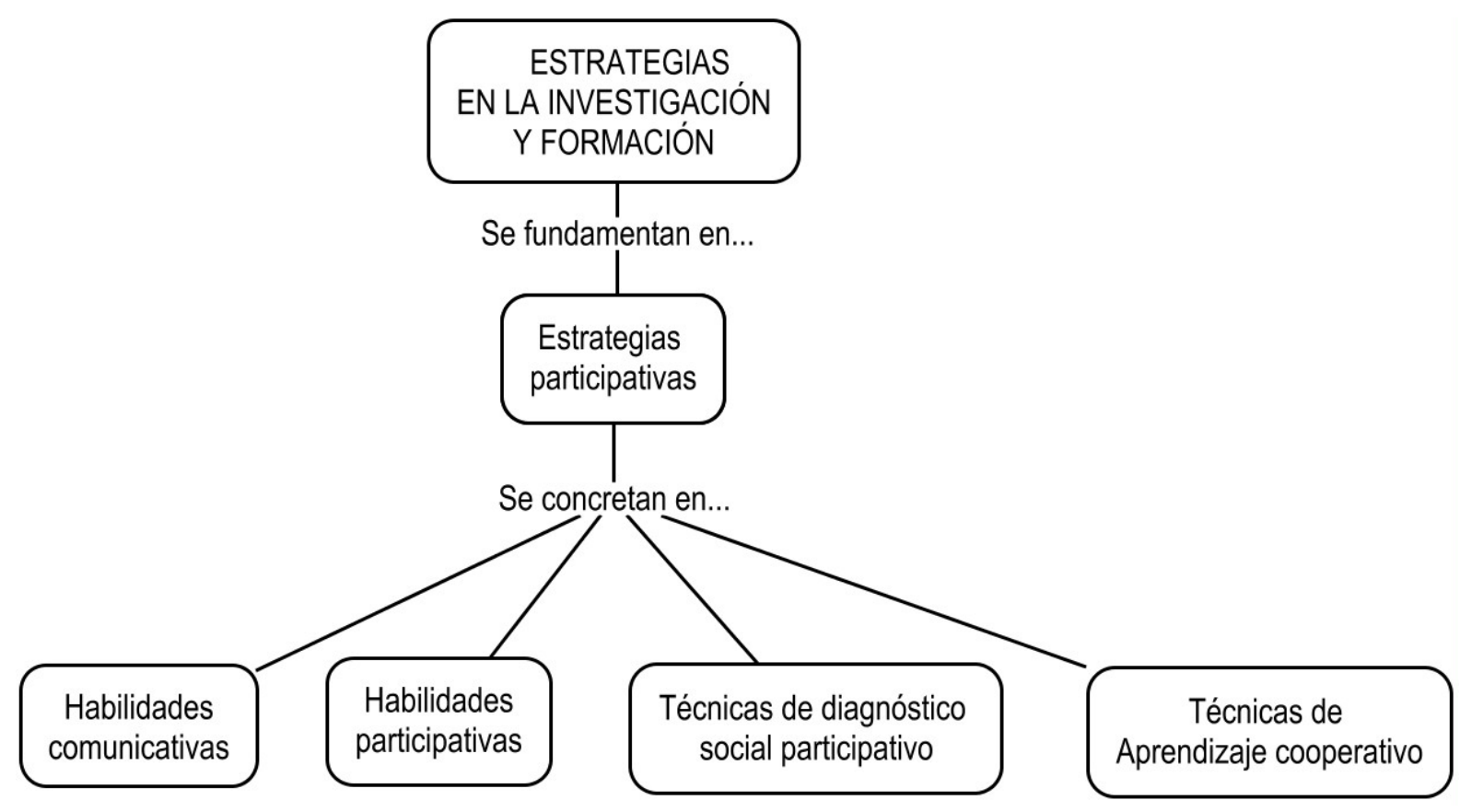

Gráfico 3. Resultados sobre las estrategias en la investigación y formación. Fuente. Elaboración propia

Respecto a la tercera pregunta, se proponen diferentes estrategias, técnicas y habilidades. Los Informantes expresan, de acuerdo a su experiencia, que la base para la MDC son las estrategias participativas, "hemos ido aprendiendo estrategias participativas para que desde el diagnóstico inicial hasta la evaluación y la sistematización de la experiencia sea accesible.” (I3, GF). La informante 2 destaca alguna de estas experiencias, "hemos hecho coanálisis de prácticas. [...]. Hemos hecho algunas cosas, como por ejemplo la difusión conjunta de resultados.” (I2, GF). Estas estrategias se concretan en "técnicas de diagnóstico social participativo y en técnicas de aprendizaje cooperativo.” (I5, R), e incorporan la comunicación como factor clave. 


\section{N V E S T I G A C I O N E S}

Así pues, ambas técnicas pueden focalizar tanto en habilidades participativas como en habilidades comunicativas, de acuerdo a las necesidades. Puesto que como dice el informante I, no es una clasificación excluyente, sino que a veces focalizan en un aspecto participativo y otras en uno comunicativo.

Las habilidades participativas ponen en valor "trabajar en estrategias el elemento de identidad, la cohesión, la identificación en el grupo, el sentimiento de pertenencia.” (I1, R). Así como, ofrecen un especial lugar al liderazgo distribuido, el empoderamiento y la participación democrática. "El empoderamiento tiene que ver con el liderazgo.” (I2, GF). Y dan buena muestra de ello:

"Por ejemplo en las estrategias de empoderamiento hay una que es vitaly es la del espejo, la de la toma de conciencia crítica. [...]. Nosotros empezamos a delegar el liderazgo de los proyectos. Lo lidera la escuela y lo lideran los maestros, nosotros estaremos acompañando. [...]. El éxito más grande ha sido en el último proyecto cuando el alumnado ha sido capaz de liderar la última fase de movilización del conocimiento en el proyecto APS, [...]" (I1, GF).

Las habilidades comunicativas proporcionan espacios de reflexión en los que se asegura la comunicación y el diálogo. "Porqué participación y comunicación van muy de la mano." (I3, GF). En algunas ocasiones estas habilidades se llevan a cabo, "por ejemplo, a partir de preguntas, introducir aquello que los hace pensar y reflexionar..." (I2, GF). Y para llevar a cabo estas estrategias se necesitan condiciones que lo faciliten.

\section{d.Condiciones limitadoras o facilitadoras para la MDC}

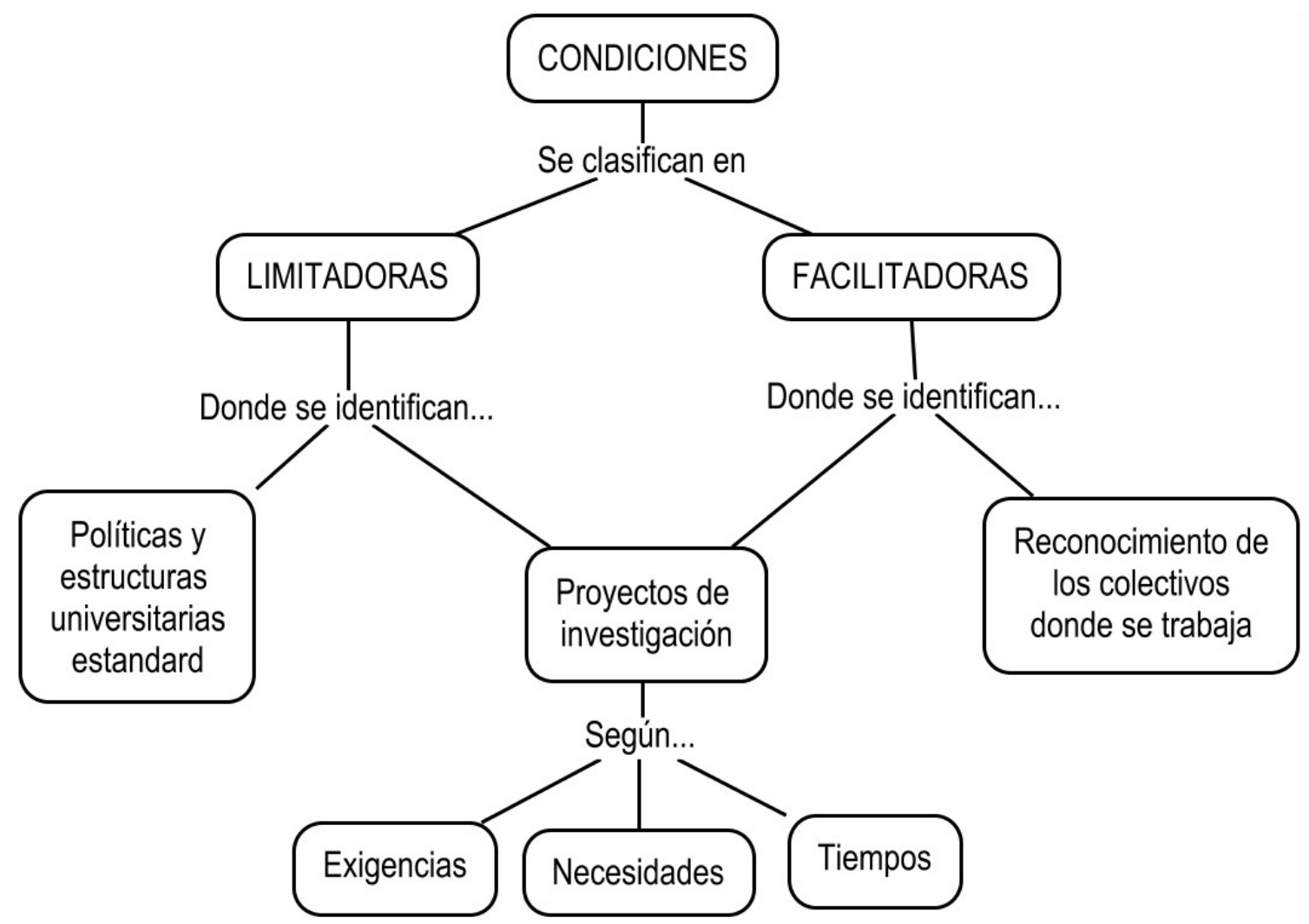

Gráfico 4. Resultados sobre las condiciones limitadoras y facilitadoras para la MDC. Fuente. Elaboración propia 


\section{N V E S T I G A C I O N E S}

Los Informantes distinguen que los proyectos pueden ser facilitadores y limitadores. "en el sentido de que tienes unos tiempos, unos plazos que cumplir y un ritmo que llevar. Esto para avanzar a veces va bien y a veces va fatal.” (I2, GF). En el propio contexto de investigación y formación los proyectos facilitan la movilización cuando los tiempos son flexibles y los recursos son accesibles. "El hecho de redactar el proyecto, releerlo y revisarlo también moviliza el conocimiento. [...], facilita que podamos aclarar conceptos.” (I2, GF).

En cuanto a las condiciones limitadoras, las políticas universitarias estándar perpetúan las relaciones jerárquicas y afectan al reconocimiento de la investigación, hecho que no favorece la MDC. "Las relaciones que se establecen en el grupo son relaciones horizontales pero las relaciones que establece la Universidad siguen siendo jerárquicas.” (I4, GF). Además, "Hay cosas estructurales y de dinámica y política universitaria que afectan al reconocimiento de este tipo de investigación. [...]. No es muy facilitador el tiempo académico para este tipo de investigación.” (I7, GF)

Respecto a las condiciones facilitadoras, destacan que el reconocimiento de los colectivos con los que se trabaja es fundamental para continuar con esta tarea. "Al final nos vale un correo que envía una persona que dice que se le ha transformado la vida y nos da las gracias por estar juntos en este proyecto. Tenemos esa satisfacción que sabemos que es la más importante.” (I1, GF)

\section{e. Estrategias, y condiciones relacionadas con el proceso intragrupal de la MDC de este grupo de investigación}

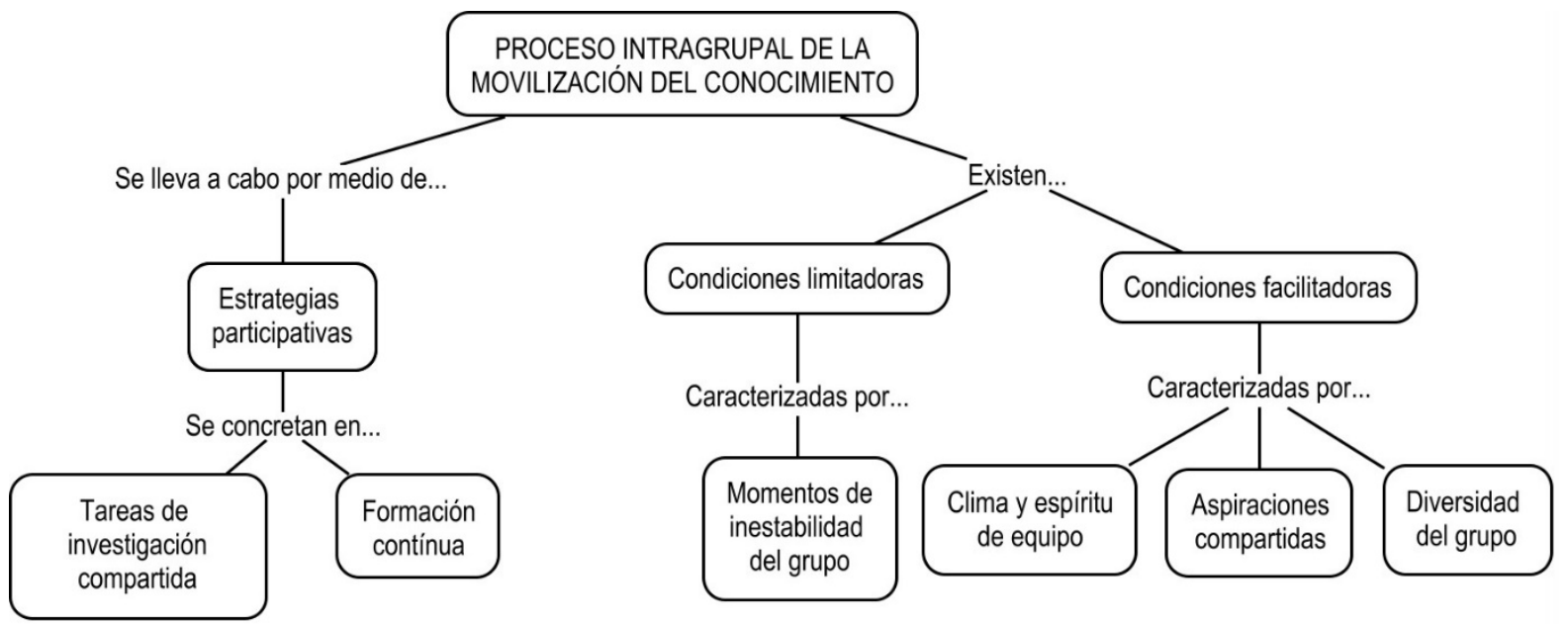

Gráfıco 5. Resultados sobre el proceso intragrupal de la movilización del conocimiento. Fuente. Elaboración propia

Respecto al proceso de MDC que se lleva a cabo de manera intragrupal se vuelven a destacar las estrategias de carácter participativo ya que: "siempre ha sido un pilar fundamental del equipo, que todo el mundo tenemos voz y tenemos alguna cosa que aportar en el proceso que estemos." (I4, GF). La Informante 3 enfatiza también en la participación de otros agentes dentro del grupo y en llevar a cabo tareas de investigación compartida. "Tener esas otras miradas es fundamental para no perder el oremus y la ética como investigadores y como personas." (I3, GF). Incluso destacan experiencias participativas mediante las cuales se ha establecido la MDC dentro del grupo. Esto ocurre con el análisis interno de las sesiones y los instrumentos utilizados para ello: 
“[...] teníamos alumnos que venían a participar y a aprender de la formación y eso generaba muchísima movilización del conocimiento y gastábamos sesiones de reunión aquí (en el seminario del departamento) para discutir qué había pasado, como habia pasado" (I4, GF).

En estos análisis destacan la reflexión como componente fundamental para la MDC, ya que les ayuda a cuestionarse y superarse en los procesos. Puesto que, "La propia estrategia del grupo no es tener una estrategia fija de reflexión, [...], las propias estrategias llevan a la reflexión continua. [...]. Son procesos muy reflexivos y muy participativos." (I7, GF). Por lo tanto, valoran las aportaciones externas y para ello a veces recurren a la formación continua, "tenemos como unas normas: tener una visión externa. En algunos momentos hemos traído gente externa para que nos hagan formación." (I2, GF).

Como condiciones limitadoras identifican los momentos de inestabilidad del grupo, "de repente somos mucha gente y por circunstancias personales o laborales tienes que salir y eso desestabiliza un poco al grupo, [...].” (I4, GF).

Por otra parte, relacionan las condiciones facilitadoras con la diversidad, las aspiraciones compartidas y el clima de equipo. Donde destacan algunas condiciones indispensables: "la primera seria cuidarnos. [...], que nos sintamos cómodos, que reconozcamos este como un lugar nuestro, que nos sintamos vinculados, que nos encontramos también desde la diferencia, [...].” (I1, GF). Cuando hablan del clima de equipo, hablan de "intentar que las aspiraciones fuesen humildes, sensatasy ajustadas a la capacidad de trabajo." (I1, GF), de los vínculos emocionales, del diálogo compartido y de las relaciones y estructuras igualitarias. Aspectos que constituyen los valores del equipo. Y respeto a la diversidad del grupo relacionan movilizar el conocimiento mediante conexiones con colaboradores externos y mediante la formación y aprendizaje continuo. Aspectos que nos recuerdan las estrategias que llevan a cabo.

De este modo, tanto el concepto de MDC, como los roles, estrategias y condicionantes quedan conectados entre sí por la visión de IAP que caracteriza este proceso.

\section{DISCUSIÓN Y CONCLUSIONES}

Una vez analizados los resultados, emanan una serie de síntesis en torno a las preguntas de investigación. Estas ideas denotan temas que podrían ser futuras líneas de investigación e intervención por parte de los investigadores en educación inclusiva.

Por un lado, respecto a la conceptualización de movilización del conocimiento, tal y como apuntaba Levin (2008), se observan dificultades para encontrar una única representación común que lo defina. Los informantes hablan de compartir, difundir, mover, conectar y construir conocimiento, pero no se alcanza una definición concreta del concepto. Sin embargo, establecen unas bases en las que coinciden y donde se sustenta la incorporación y uso de este concepto en sus propios discursos y prácticas. Parece una necesidad del grupo atajar el término desde el paradigma de la IAP para conectar con sus experiencias, empoderarse y explicar cómo lo llevan a cabo.

Para definir el concepto, el grupo parte de situar la naturaleza de la MDC desde un paradigma concreto: la IAP (Ander-Egg, 2003), donde se dota de sentido y se establece una relación directa 
con todo lo que este paradigma conlleva: educación, investigación y sociedad inclusiva. Una vez establecido el punto de partida, relacionan las prácticas que ellos han llevado a cabo, en las que identifican este proceso. Para continuar ofreciendo más detalles que componen la MDC, como son: la construcción compartida de conocimiento, el empoderamiento o la transformación de realidades.

Además, se diferencia la transferencia de conocimiento de la MDC. La primera se entiende como una mera acción de recopilación y exposición y la MDC como un proceso accesible, democrático y que se desarrolla en cualquier momento para transformar la sociedad. La MDC es el resultado de estrategias de trabajo en red, intercambio y valoración (difusión y transferencia).

A pesar de no establecer una definición concreta, se van incorporando los elementos que la constituyen desde la visión del grupo. La MDC se intuye como un término relevante que se empieza a utilizar para explicar muchas de las acciones que llevan a cabo o a las que aspira el grupo. Compartir esto les ha ayudado a ubicarse en el término y a poner nombre a aquello en lo que ya se sentían partícipes. La MDC no solo les proporciona una nomenclatura sino también una fundamentación donde identifican matices que van surgiendo durante el proceso de investigación.

Cabe destacar, que compartir experiencias referentes a la MDC con otros investigadores (como han expresado que hicieron con investigadores de Canadá) también les ha facilitado esclarecer e incorporar el concepto en su discurso y en su manera de hacer. Puesto que, "a medida que los equipos creaban y compartían el conocimiento con otros en diferentes niveles de la organización, el conocimiento se amplificaba y movilizaba a medida que avanzaba a través de individuos, grupos, divisiones y organizaciones” (Reid, 2014, p. 333).

En este sentido se valora positivamente la apropiación del concepto dentro del grupo, pero se siente la necesidad de constituir un marco teórico que alumbre y facilite los medios para designar con precisión y rigurosidad una definición concreta. Puesto que si designamos un concepto partiendo únicamente de las propias vivencias podemos estar corriendo varios riesgos: solapar significados (Levin, 2008), poner al mismo nivel diferentes conceptos que requieren de especificaciones (como MDC y Educación Inclusiva), encasillar el término dentro de un único contexto, o desconocer parte de la identidad de un concepto; que solo nos la puede ofrecer un análisis contrastado y compartido del mismo a través de la literatura. Por eso, consideramos necesario compartir una formación orientada a la importancia del MDC y su terminología, así como de los procesos, estrategias y movimientos, de acuerdo con Perines (2017).

Respecto a los roles asumidos por los investigadores, se puede observar la importancia de definir roles en los procesos de investigación, ya que afectan a la manera de actuar y llevar a cabo cualquier procedimiento. Así pues, quedan visibilizados y diferenciados siete de ellos, de acuerdo a las prácticas narradas por los Informantes. En ellos se diferencian el rol de experto y el de acompañante con diferencias sustanciales, comentadas en los resultados. El resto de roles comparten rasgos comunes y se delimitan dentro del rol de acompañante. Esto ocurre con los roles de: dinamizador, mediador, catalizador, amigo crítico y coinvestigador.

En este sentido, se encuentran coincidencias entre los roles que identifican los informantes y los roles que aparecen en la literatura (Moliner y St-Vicent, 2014). Por ejemplo, entre el rol de ex- 
perto (nombrado por los informantes) con el de investigador profesional (Dolbecy Prud'homme, 2009). Ya que todas estas denominaciones coinciden en esencia con un carácter más externo e instruccional. Así como el de acompañante (nombrado por los informantes) con el de amigo crítico (Both y Ainscow, 2002), facilitador de recursos (Wang y Mu, 2013), participante, observador, facilitador del trabajo cooperativo y agente de cambio. Puesto que estas denominaciones están conectadas con una acción más participativa y activa en el contexto.

Además, se incorpora uno más, el de coinvestigador. De acuerdo con Valenzuela (2008) es un rol que ofrece una mirada más abierta y crítica, implicando a toda la comunidad, en el transcurso del proyecto. De este modo y de acuerdo a las aspiraciones que expresan los informantes, este sería el rol propiamente objeto de la MDC, puesto que es el rol que más se identifica con el trabajo "mano a mano" con todos los agentes, para compartir los saberes en todo momento. Aunque este rol despierta inquietudes dentro del grupo: ¿Ser coinvestigador implica coinvestigar en todas las fases? ¿Se está llevando a cabo en el grupo el rol de coinvestigador? ¿Con qué intensidad y frecuencia?

Por tanto, en futuras investigaciones, proponemos formalizar una tabla o matriz de consulta que concrete y categorice los roles relacionados con la MDC, de acuerdo a las exigencias, necesidades y tiempos. Ya que, en estos momentos, aún resulta complejo crear un diagrama delimitador que contenga los condicionantes expresados por los informantes.

Por otro lado, en este estudio se observa que las estrategias y técnicas que generan esa movilización son modos de trabajo que tienen en cuenta a todas las voces desde la participación comunitaria (Traver, Sales y Moliner, 2010) y democrática, la cooperación y la inclusión de todos los agentes. La comunicación es la base para lograr el empoderamiento que capacita a la sociedad para compartir todos sus saberes y hacer de ellos algo suyo. De acuerdo con Dewey (1995): "La comunicación es un proceso a compartir la experiencia hasta que ésta se convierte en una posesión común” (p.20).

En este sentido, el equipo de investigación tiene una gran responsabilidad en cuanto al diseño y aplicación de estrategias que trabajan por, para y desde la MDC. Y por ello, los informantes reconocen la vinculación de estas estrategias con modelos e instrumentos de mejora y transformación de centros educativos como son el Index for inclusión y la Guía CEIN - Guía para la construcción de la escuela intercultural inclusiva, (Booth y Ainscow, 2002; Traver, Sales y Moliner, 2010).

Gracias a este análisis, se extrae que el grupo de investigación se beneficia y utiliza las estrategias de formación como estrategias de investigación. Pero sus acciones como grupo de investigación están interconectadas con las de formación, aunque aparentemente parezcan dos ámbitos diferentes. Así pues, se hace necesaria una sinergia entre investigación y formación. En ella se justifica que muchas de sus respuestas, en referencias a su modus operandi, sean tomadas desde la visión investigadora.

El fin último que se persigue desde estos procesos, tanto de investigación como de formación, es el diseño de maneras de planificación conjunta: la MDC no como una fase final, sino durante el proceso. Y en este sentido, de acuerdo con Traver, Sales y Moliner (2010) las estrategias de liderazgo distribuido deberían ser un objetivo compartido que de luz y guíe la MDC. 
De acuerdo con las aportaciones analizadas, estos procesos quedan limitados en gran medida por las restricciones temporales que caracterizan este tipo de investigación. Ante esto es esencial una llamada de atención a las infraestructuras burocráticas, la administración encargada de dotar de ayudas a las investigaciones, para crear facilidades para cualquiera de los agentes que participan en los estudios. Es decir, se debe trabajar para atajar las dificultades en cuanto a plazos o incluso a la inexistencia de espacios temporales para la investigación. A través de los tiempos calmados para lograr avances sociales. Y para ello, se necesitan tiempos para generar y compartir inquietudes y conocimiento en todos los sectores y contextos. En este sentido coincidimos con otro estudio previo de la misma índole como es el de Moliner y Sánchez, (2019).

Del mismo modo, también existen facilitadores como las interacciones entre diferentes instituciones: universidad, escuela u organizaciones locales (Ainscow, 2004). Así como el propio reconocimiento de los participantes y las redes humanas que se forman y se mantienen para conseguir los objetivos comunes. En esencia, aquí se encuentra la bocanada de aire fresco que se necesita para continuar progresando hacia la inclusión.

En definitiva, la dirección es clara, las prácticas y estrategias empleadas para la MDC van hacia la misma línea y los condicionantes quedan definidos. Aunque las estrategias, condicionantes y relaciones que se viven de manera intragrupal son un aspecto poco tratado, a la vez que iluminador, dentro de este estudio de la MDC, tal y como comenta Parrilla (2009). De manera intragrupal se experimentan y fundamentan conceptos de los que se empodera el grupo y donde se constituyen la identidad como equipo de investigación en un paradigma específico de investigación. Es importante hacer cuestionamientos para comprender cómo se llevan a cabo sus tareas, para avanzar y no estancarse. Pero no solo fijarnos en las metodologías de trabajo, sino ir más allá. Ya que este tipo de investigación, con mayor característica humanística, dota de poder la evaluación antropológica y relacional para comprender aquellos aspectos que luego pueden afectar y encaminar las acciones de las investigaciones en el campo.

Por ello creemos importante un análisis interno dentro de los grupos de investigación. Tanto para la valoración como para la mejora. ¿Un grupo de investigación en investigación inclusiva es inclusivo? ¿Qué roles se establecen dentro de los propios grupos de investigación? ¿Qué estrategias? ¿Qué relaciones humanas y sociales o jerarquías, se establecen? ¿Qué delimita o facilita sus tareas? ¿Cómo afecta esto a la MDC? Estos cuestionamientos los dejamos para una futura línea de investigación. Pero era importante tratarlos dentro de este estudio, aunque solo fuese de un modo incipiente.

En este sentido, se identifican los momentos de inestabilidad como dificultades para la MDC dentro del grupo; y el diálogo, la formación continua y la diversidad del grupo, como algunos aspectos facilitadores. Por eso, también es un factor clave estudiar donde se inicia la MDC, si dentro del propio grupo o en el propio entorno de la investigación.

Así pues, desde este estudio defendemos que los investigadores en educación inclusiva deben centrar los procesos para la MDC llevando a cabo estrategias que aseguren la participación de toda la sociedad, por medio de un trabajo inclusivo, cooperativo, crítico y democrático del grupo. 


\section{N V E S T I G A C I O N E S}

\section{REFERENCIAS}

Ainscow, M. (2004). El desarrollo de sistemas educativos inclusivos: ¿Cuáles son las palancas de cambio? Journal of educational change, 5(4), 1-20. URL https://bit.ly/380tjvv

Ander-Egg, E. (2003). Repensando la investigación-acción participativa. Lumen Hvmanitas.

Barrio, J.L. (2009). Hacia una educación inclusiva para todos. Revista Complutense de Educación, 1(20), 13-31. URL https://bit.ly/3oJmJjI

Bisquerra, R. (2004). Metodología de la investigación educativa. La muralla.

Bonilla-Jiménez, F. I., y Escobar,J. (2017). Grupos focales: una guía conceptual y metodológica. Cuadernos hispanoamericanos de psicología, 9(1), 51-67.

Booth, T., y Ainscow, M. (2002). Guía para la evaluación y mejora de la educación inclusiva. Consorcio Universitario para la Educación Inclusiva. Universidad Autónoma de Madrid, 16.

Dewey, J. (1995). Democracia y educación. Ediciones Morata.

Dolbec, A. \& Prud'homme, L. (2009). La recherche-action. En B. Gauthier (Dir.), La recherche sociale: de la problématique à la collecte des données (pp. 531-569). Presses de l’Université du Québec.

Echeita, G. (2017). Educación Inclusiva. Sonrisas y lágrimas. Aula Abierta, 46, 17-24. URL https://bit. ly/2K $4 \mathrm{KpOH}$

Fischman, G. E., Anderson, K. T., Tefera, A. A., \& Zuiker, S. J. (2018). If mobilizing educational research is the answer, who can afford to ask the question? An analysis of faculty perspectives on knowledge mobilization for scholarship in education. AERA Open, 4(1), 1-17. URL https://bit.ly/3mgK5LI

Landry, R., Becheikh, N., Amara, N., Ziam, S., Idrissi, O., \& Castonguay, Y. (2008). Revue systématique des écrits sur le transfert de connaissances en éducation. Québec.

Levin, B. (2008, May). Thinking about knowledge mobilization. In an invitational symposium sponsored by the Canadian Council on Learning and the Social Sciences and Humanities Research Council of Canada (pp.15-18).

Moliner, O., y Ramel, S. (2018). Una mirada sobre el trabajo en red del laboratoire international sur l'inclusion scolaire (LISIS): Afianzando una cultura colaborativa para la movilización del conocimiento sobre educación inclusiva. Profesorado, Revista de Currículum y Formación del Profesorado, 22(2), 91-109.

Moliner, O., y Sánchez, T. (2019). Reconstruyendo la figura del asesor en educación inclusiva: Estudio de los procesos de movilización del conocimiento en la formación continua del profesorado. Revista de Educación Inclusiva, 12(1),291-310.

Moliner, O., y St-Vincent, L. A. (2014). Dilemas éticos de los investigadores que acompañan procesos de investigación-acción en el marco de la escuela intercultural inclusiva. Revista latinoamericana de educación inclusiva, 8(2), 49-68.

Parrilla, Á. (2009). ¿Y si la investigación sobre inclusión no fuera inclusiva? Reflexiones desde una investigación biográfico-narrativa. Revista de educación, 349, 101-117.

Parrilla, Á., Raposo-Rivas, M. y Martínez-Figueira, M.E. (2016). Procesos de movilización y comunicación del conocimiento en la investigación participativa. Opción: Revista de Ciencias Humanas y Sociales, (12), 1166-1187. 


\section{N V E S T I G A C I O N E S}

Pérez, R., García, O., y Ortíz, V. (2016). La movilización del conocimiento para la innovación social. Revista Electrónica Pesquiseduca, 8(16), 277-294.

Perines, H. (2017). Movilización del conocimiento en educación. Conexión entre la investigación, la política y la práctica: una aproximación teórica. Páginas de Educación, 10(1), 137-150.

Reid, S. (2014). Knowledge influencers: leaders influencing knowledge creation and mobilization.Journal of Educational Administration, 52(3), 332-357. DOI 10.1108/JEA-01-2013-0013

Social Science and Humanities Research Council of Canada. (2018). Guidelines for effective knowledge mobilization.

Traver, J., Sales, A., y Moliner, O. (2010). Ampliando el territorio: algunas claves sobre la participación de la comunidad educativa. REICE: Revista Iberoamericana sobre Calidad, Eficacia y Cambio en Educación, 8(3), 96-119.

UQAM. (31 de marzo de 2018). Mobilisation des connaissances. UQAM. URL https://bit.ly/34a6Evm

Valenzuela,C.E.(2008). Coinvestigación: organizaciones popularesy nuevas prácticas de saber.Nómadas, (29), 112-127.

Wang, Q., \& Mu, H. (2013). The roles of university researchers in a University-school collaborative action research project-A Chinese experience. Multidisciplinary Journal of Educational Research, 3(2), 101129. 